\title{
Analysis of Time Series Data Using Maximal Overlap Discrete Wavelet Transform Autoregressive Moving Average
}

\author{
Sella Nofriska Sudrimo ${ }^{1}$, Kusman Sadik $^{2}$, I Made Sumertajaya ${ }^{3}$ \\ \{sellans98@gmail.com, kusmansadik@gmail.com, imsjaya.stk@gmail.com\} \\ Student of Department of Statistics, IPB University, Indonesia ${ }^{1}$, \\ Lecturer of Department of Statistics, IPB University, Indonesia ${ }^{2,3}$
}

\begin{abstract}
The price of broiler chickens has fluctuations pattern or certain wave patterns. This study aims to predict broiler chicken price data that have to fluctuate and nonstationary using MODWT-ARMA models andARIMA models and also see the ability of MODWT-ARMA in increasing accuracy in predicting data. In this study, the data is separated using wavelet transforms namely MODWT into two-part is wavelet and smooth signal, then each signal is modeled using the ARMA model and the final of the process is to recombine all signals. The results show that the MODWT-ARMA model has a smaller RMSE and normalized error than the ARIMA which is 1175.97 and 0.68 for the MODWT-ARMA model while 2365.85 and 2.77 for the ARIMA model. The conclusion in this study, MODWT-ARMA can handle broiler chicken price data in Bogor better than the ARIMA model and can improve the accuracy of prediction results.
\end{abstract}

Keywords: Autoregressive moving average, Discrete wavelet transform, Maximal overlap discrete wavelet transform

\section{Introduction}

Broiler chicken price data has a fluctuation or wave pattern (forming valleys and peaks) so that usually the plot formed by broiler chicken price data is not smooth. Although fluctuation or waves pattern in chicken prices is not too sharp, it can affect traders in the market. The price of broiler chickens that often experience fluctuations or certain waves and sometimes have an upward trend every day makes the price of chickens need to be observed every day. The analysis of time series data aims to obtain an appropriate model so that it can predict data for some time to come with the time series model formed is inseparable from the influence of previous times. One method that is often used to handle time-series data that has fluctuating patterns and non-stationary is the maximal overlap discrete wavelet transform autoregressive moving average (MODWT-ARMA) method.

MODWT-ARMA is a hybrid model of the MODWT model and the ARMA model that deals with non-stationary time-series data [5]. ARIMA method is a time-series data method that can handle data that is not stationary. Wavelet has the meaning of a small wave that can separate the signal into two components, namely the scale and detail, the purpose of which is to transform the signal so that it is easy to understand. In addition, wavelets are also able to represent functions that are not smooth or functions with surges or have high volatility[5]. 
Wavelet transformation method is divided into two, namely continuous wavelet transformation (CWT) and discrete wavelet transformation (DWT). Wavelet transforms that is often applied to handle time-series data that is observed in daily, weekly, monthly, yearly is DWT. DWT is widely used for time series data transformation that has high volatility such as rainfall data, stock data, inflation data, and other data that are not stationary and difficult to be modeled if only using the time series model in general.

The weakness of DWT is that this method can only be used for a certain amount of data. The DWT method can only be applied to data with the same amount of data with a multiple of two so that the signal will be truncated if the amount of data is not suited and causes a lot of information to be lost.MODWT is a modification of the DWT method that can cover all data that has a random amount. The advantage of the MODWT is able to decompose data by breaking the data into two parts with each level in the decomposition having wavelet and smooth coefficients as much as the length of the data.

Some studies had shown that MODWT-ARMA was more accurate in modeling nonstationary and fluctuating data than the ARIMA method. This study discusses the analysis of daily broiler chicken price data in Bogor in 2018 using the MODWT-ARMA method. The MODWT-ARMA method is used because the price of broiler chickens in 2018 in Bogor has a fluctuating pattern and is not stationary. This study aims to predict broiler chicken price data that have to fluctuate and non-stationary using MODWT-ARMA models and ARIMA models and compare MODWT-ARMA model with ARIMA models to see the ability of MODWTARMA in increasing accuracy in predicting data.

\section{Materials}

The data used in this study are secondary data from the Indonesian People's Poultry Association (PINSAR Indonesia). The data is the daily broiler chicken price data in Bogor in 2018. The amount of data is 365 data with the unit price used is Rupiah per kilogram. The price of chicken determined by PINSAR Indonesia is a price that is a benchmark for prices in regions throughout Indonesia.

\section{Method}

Data analysis was performed using R 3.5.2 software with the following steps:

1. Dividing data into training data and test data. The amount of training data as much as $90 \%$ of the research data is 328 data with the data used is the first day data until the 328th day data while the amount of test data is $10 \%$ of the research data that is 37 data with the data used is the 329th day data until the day data to 365th. In this study, training data is used to build the model and test data is used to validate the model.

2. Exploring training data to see an overview of broiler chicken price data in Bogor 2018 with descriptive statistics

3. Modeling using MODWT-ARMA. The steps are as follows:

a. Calculating MODWT wavelet filters $\left(\widetilde{\mathrm{h}}_{\mathrm{j}, 1}\right)$ and smooth filters $\left(\widetilde{\mathrm{g}}_{\mathrm{j}, \mathrm{l}}\right) \mathrm{using}$ Daubechies 4 wavelet transforms with $\mathrm{J}=4$ with multi-resolution analysis techniques (MRA ). Next is sharing the filter with Daubechies 4 [17]

Filter smooth: $\mathrm{g}_{\mathrm{j}, 0}=\frac{1+(3)^{\frac{1}{2}}}{4(2)^{\frac{1}{2}}}, \mathrm{~g}_{\mathrm{j}, 1}=\frac{3+(3)^{\frac{1}{2}}}{4(2)^{\frac{1}{2}}}, \mathrm{~g}_{\mathrm{j}, 2}=\frac{3-(3)^{\frac{1}{2}}}{4(2)^{\frac{1}{2}}}, \mathrm{~g}_{\mathrm{j}, 3}=\frac{1-(3)^{\frac{1}{2}}}{4(2)^{\frac{1}{2}}}$ 
Filter wavelet $: \mathrm{h}_{\mathrm{j}, 0}=\frac{1-(3)^{\frac{1}{2}}}{4(2)^{\frac{1}{2}}}, \mathrm{~h}_{\mathrm{j}, 1}=\frac{-3+(3)^{\frac{1}{2}}}{4(2)^{\frac{1}{2}}}, \mathrm{~h}_{\mathrm{j}, 2}=\frac{3+(3))^{\frac{1}{2}}}{4(2)^{\frac{1}{2}}}, \mathrm{~h}_{\mathrm{j}, 3}=\frac{-1-(3)^{\frac{1}{2}}}{4(2)^{\frac{1}{2}}}$

with $g_{l}$ is a smooth filter of DWT dan $h_{l}$ is a wavelet filter DWTof DWT [11]. While the wavelet filter of MODWT $\left(\tilde{\mathrm{h}}_{\mathrm{j}, 1}\right)$ using formula $\tilde{h}_{j, l}=\frac{h_{j, l}}{2^{j / 2}}$ and smoothfilter $\left(\tilde{\mathrm{g}}_{\mathrm{j}, \mathrm{l}}\right)$ using formula $\tilde{g}_{j, l}=\frac{g_{j, l}}{2^{j / 2}}$

b. Calculating the MODWT wavelet coefficient $\left(\widetilde{\mathrm{W}}_{\mathrm{j}}\right)$ using formula $\widetilde{\mathrm{W}}_{\mathrm{j}}=\widetilde{\boldsymbol{\omega}}_{\mathrm{j}}$ Xand the smooth coefficient $\left(\widetilde{\mathrm{V}}_{\mathrm{j}}\right)$ using formula $\widetilde{\mathrm{V}}_{\mathrm{j}}=\widetilde{\mathbf{v}}_{\mathrm{j}} \mathrm{X}$

c. Determining the MODWT wavelet signal $\left(\widetilde{\mathrm{D}}_{\mathrm{j}, \mathrm{t}}\right)$ using formula:

and smooth signal $\left(\tilde{\mathrm{S}}_{\mathrm{J}, \mathrm{t}}\right)$ using formula:

$$
\widetilde{\mathrm{D}}_{\mathrm{j}, \mathrm{t}}=\left|\varphi_{\mathrm{j}}(\mathrm{B})-1\right| \widetilde{\mathrm{D}}_{\mathrm{j}, \mathrm{t}}+\theta_{\mathrm{j}}(\mathrm{B}) \varepsilon_{\mathrm{t}}
$$

$$
\tilde{\mathrm{S}}_{\mathrm{J}, \mathrm{t}}=|\varphi(\mathrm{B})-1| \tilde{\mathrm{S}}_{\mathrm{J}, \mathrm{t}}+\theta(\mathrm{B}) \varepsilon_{\mathrm{t}}
$$

d. Determining the order $p, d$, q for the ARMA model (p, d, q) of each level of wavelet and smooth signal to produce the coefficients $\varphi$ and $\theta$.In modeling, there are several steps including:

1. Making an ACF plot and calculate the Augmented Dickey-Fuller test statistics (ADF) to see if the data is stationary. If the data is not stationary, carried out differencing to stationary data.

2. Determining candidate ARIMA models with ACF plots, PACF plots, and ESACF. Next, determine the overfitting of the model obtained.

3. Testing the goodness of the model to determine the best ARIMA (p, d, q) model with the auto.arima function using $\mathrm{R}$ software with "forecast" packages. In determining the best ARIMA model ( $\mathrm{p}, \mathrm{d}, \mathrm{q})$, there are several criteria for the goodness of the model, consist of:

a) Akaike Information Criterion (AIC)

$$
\begin{array}{lcc}
\mathrm{AIC}=-2 \ln (\mathrm{L})+2 \mathrm{k} \\
\text { mith } \mathcal{m} \text { adalah }
\end{array} \quad \begin{gathered}
(5) \\
\text { function, }
\end{gathered}
$$

$\mathrm{S}(\phi, \mu, \theta)=\sum_{\mathrm{t}} \mathrm{e}_{\mathrm{t}}(\phi, \mu, \theta)$ and $\hat{\sigma}_{\mathrm{e}}^{2}=\mathrm{S}(\hat{\phi}, \hat{\mu}, \hat{\theta}) / \mathrm{n}$, and $\mathrm{k}$ is the number of parameters in

the model. The best model is the model that has the smallest AIC value [19].

b) Akaike's Information Criterion Bias Corrected (AICc)

$$
\mathrm{AICc}=-2 \ln (\mathrm{L})+2 \mathrm{k}\left(\frac{\mathrm{n}}{\mathrm{n}-\mathrm{k}-1}\right)
$$

with the best model obtained based on the smallest AICc value [3].

c) Bayesian Information Criterion (BIC)

$$
\mathrm{BIC}=-2 \ln (\mathrm{L})+\mathrm{k} \ln (\mathrm{n})
$$

where $\mathrm{n}$ is the number of observations and $\mathrm{k}$ is the number of parameters. The best model is the model that has the smallest BIC value [8].

e. Entering the coefficient values $\varphi$ and $\theta$ in $\widetilde{\mathrm{S}}_{\mathrm{J}, \mathrm{N}}$ using equation (4) and $\widetilde{\mathrm{D}}_{\mathrm{j}, \mathrm{N}}$ using equation (3)as a weighting in the MODWT-ARMA model.

f. Modeling using the MODWT-ARMA model

$$
\hat{Y}_{t}=\sum_{j=1}^{J} \widehat{D}_{j, t}+\hat{S}_{J, t}
$$

4. Evaluate the goodness of the model based on RMSE [4] and normalized error [20]. 


$$
\begin{aligned}
& \operatorname{RMSE}=\left(\frac{1}{N} \sum_{\mathrm{i}=1}^{\mathrm{n}}\left(\mathrm{y}_{\mathrm{t}}-\hat{\mathrm{y}}_{\mathrm{t}}\right)^{2}\right)^{\frac{1}{2}} \\
& \mathrm{Q}=\frac{\sum_{\mathrm{t}=1}^{\mathrm{t} \text { test }}\left(\mathrm{Y}_{\mathrm{t}}-\widehat{\mathrm{Y}}_{\mathrm{t}}\right)^{2}}{\sum_{\mathrm{t}=1}^{\text {test }}\left(\mathrm{Y}_{\mathrm{t}}-\overline{\mathrm{Y}}_{\mathrm{t}}\right)^{2}}
\end{aligned}
$$

where $\mathrm{n}$ is the amount of data, $y_{\mathrm{t}}$ is the price of boiler chicken at time $\mathrm{t}, \hat{\mathrm{y}}_{\mathrm{t}}$ is the estimated data of boiler chicken price at time $t$ and $Q$ is normalized error.

\section{Result and Discussion}

\subsection{Exploring Data}

At this stage, data exploration was carried out to see the characteristics of broiler chicken price data in the Regency and City of Bogor in 2018, which were presented in Figure 1. The figure showed that the data formed a fluctuating pattern

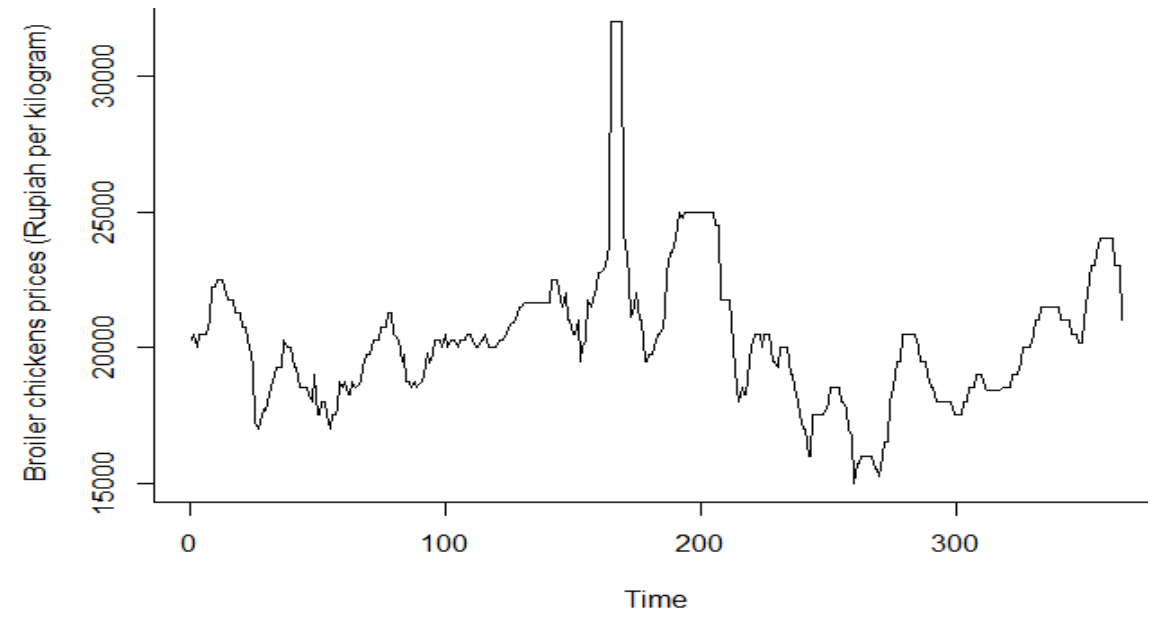

Fig. 1.The development of broiler chicken prices in Bogor in 2018

Figure 1 showed that the price of boiler chicken in Bogor in 2018 had an extreme or outlier value. Outliers in time series data have a special way of detecting them. Figure 2 showed the ACF movement decreases slowly (tail of slowly). The ACF plot that gone down slowly showed that the data was non-stationary. Next was the Augmented Dickey-Fuller test (ADF) to see the stationary data with the null hypothesis in the form of data containing unitroots (non-stationary data) and alternative hypotheses of data not containing unit-roots (stationary data).

The results of the statistical data stationary test using the ADF test showed a DickeyFuller t-value of -3.25 with a p-value of 0.09. The p-value obtained from the ADF test was greater than 0.05 so it did not support rejecting Ho. That was, the data used in this study had unit-roots or non-stationary data. As with the results shown in the ACF plot (Figure 2), the ADF test also showed the same conclusion that the data was not stationary. In general, timeseries data that was not stationary needs to be done differencing so that it became stationary. 
After exploring the data, the next step was modeling the data using the MODWT-ARMA model.

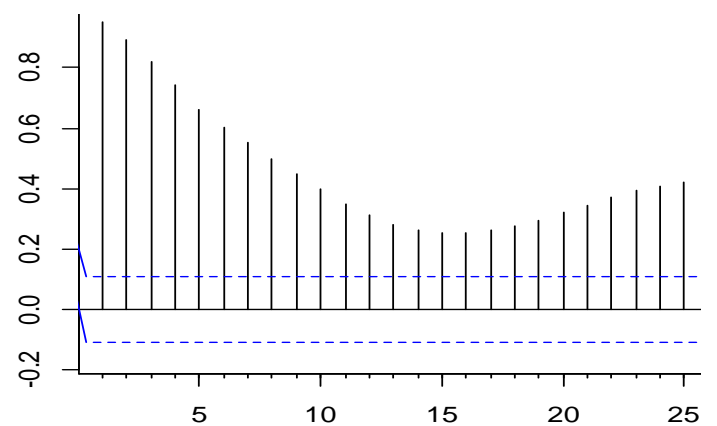

Fig. 2.Plot ACF of daily broiler chicken price data in Bogor in 2018

\subsection{Modeling Using MODWT-ARMA}

The next step is modeling the data using the MODWT-ARMA model. Modeling using MODWT-ARMA in this study can be done by decomposing the data into several signal levels using MODWT, modeling each signal of MODWT, modeling signals with selected model candidates using the ARMA model, and modeling using MODWT-ARMA by combining all signals that have been modeled.

\section{Decomposing The Data Into Several Signal Levels Using MODWT}

This study compares the Haar and Daubechies wavelets with multi-resolution filtering (MRA) filtering techniques. The reason for using the Haar wavelet was because the Haar wavelet was the oldest wavelet and used the simplest technique in data decomposition, while the using Daubechies because Daubechies was the wavelet type that provides the highest signal recognition level compared to Symlets and Coiflets [1].

Table 1. Evaluation of wavelet types at the number of different levels

\begin{tabular}{lccc}
\hline Type & $\begin{array}{c}\text { The Number } \\
\text { of Level }\end{array}$ & RMSE & Q \\
\hline Haar & 1 & 2614.68 & 3.38 \\
& 2 & 3006.26 & 4.47 \\
& 3 & 2201.16 & 2.40 \\
& 4 & 1864.86 & 1.72 \\
& 5 & 1872.39 & 1.74 \\
& 6 & 2113.90 & 2.21 \\
Daubechies 4 & 7 & 2112.26 & 2.21 \\
& 8 & 2111.49 & 2.21 \\
& 1 & 2199.46 & 2.39 \\
& 2 & 2561.49 & 3.25 \\
& 3 & 1621.97 & 1.29 \\
& 4 & 1211.72 & 0.73 \\
& 5 & 1661.19 & 1.37
\end{tabular}




\begin{tabular}{cccc}
\hline Type & $\begin{array}{l}\text { The Number } \\
\text { of Level }\end{array}$ & RMSE & Q \\
\hline 6 & 2548.43 & 3.21 \\
7 & 2548.52 & 3.21 \\
8 & 2547.90 & 3.21 \\
\hline
\end{tabular}

Table 1 showed that Haar and Daubechies 4 wavelets had the lowest RMSE and Q values at level 4. In Table 1 it could be seen that the data decomposition using Daubechies 4 wavelets with 4 levels had RMSE and Q values the smallest was 1211.72 and 0.73 . The Haar Wavelet had the smallest RMSE and Q values of 1864.86 and 1.72 at the number of level 4 . Based on the results in Table 1, the Daubechies 4 wavelet had a smaller RMSE and Q value than the Haar wavelet. So in this study, the data decomposition used Daubechies 4 wavelet with many levels is $4(\mathrm{~J}=4)$ used for broiler chicken price data in Bogor in 2018.

Table 2. Waveletcoiffecient

\begin{tabular}{lrrrr}
\hline Time & W1 & W2 & W3 & W4 \\
\hline 1 & 193.63 & 66.13 & 265.95 & -451.80 \\
2 & -6.13 & 133.22 & 361.96 & -355.39 \\
3 & -272.88 & 229.48 & 438.28 & -253.04 \\
. &. &. &. &. \\
. &. &. &. &. \\
. &. &. &. &. \\
326 & -170.75 & -92.69 & -147.20 & 90.77 \\
327 & 45.75 & 31.25 & -118.54 & 188.84 \\
328 & 170.75 & -57.19 & -136.94 & 262.37 \\
\hline
\end{tabular}

Table 3.Smooth coiffecient

\begin{tabular}{ccccc}
\hline Time & V1 & V2 & V3 & V4 \\
\hline 1 & 20777.36 & 21253.22 & 21505.86 & 20723.12 \\
2 & 20339.86 & 21002.80 & 21436.04 & 20858.92 \\
3 & 20243.87 & 20643.58 & 21305.91 & 20969.89 \\
$\cdot$ & $\cdot$ &. &. &. \\
. &. &. &. &. \\
. &. &. &. &. \\
326 & 19637.26 & 19237.42 & 18770.06 & 18670.50 \\
327 & 20012.26 & 19487.14 & 18935.68 & 18709.26 \\
328 & 20045.75 & 19771.45 & 19115.62 & 18763.63 \\
\hline
\end{tabular}

The next stage, data was separated with Daubechies 4 wavelet $(1=1,2,3,4)$ where 1 was the filter length whose length matches the type of wavelet used. The results of the data decomposition obtained $D_{j}$ and $S_{J}$ signals. The $D_{j}$ signal was a detail/wavelet signal with $j=1$, ..., $\mathrm{J}$ while $\mathrm{S}_{\mathrm{J}}$ was the smooth signal of $\mathrm{Jth}$. Before the wavelet signal and smooth signal were obtained, the wavelet filter value $\left(\tilde{\mathrm{h}}_{\mathrm{j}, 1}\right)$ and smooth filter were calculated $\left(\widetilde{\mathrm{g}}_{\mathrm{j}, 1}\right)$ as well as the wavelet coefficient and smooth coefficient first. The results of calculations using the wavelet function showed that at the first level the value of the first wavelet filter was -0.09 , the second wavelet filter was -0.16 , the third wavelet filter was 0.59 , and the fourth wavelet filter was 0.34. Whereas at the same level, the first smooth filter was 0.34 , the second smooth filter was 
0.59 , the third smooth filter was 0.16 , and the fourth smooth filter was -0.09 . Next, calculate the wavelet coefficient and smooth coefficient.

Table 2 showed the wavelet coefficients at each level of decomposition. Wavelet coefficient symbolized by $\mathrm{Wj}$ with $\mathrm{j}$ is the number of levels used. Wavelet coefficient value was the result of subtracting the $\mathrm{t}$-data from one time before $(\mathrm{t}-1)$ divided by the two roots so that the resulting values are negative. These results were different from the smooth coefficient. The smooth coefficient was derived from the sum of the $\mathrm{t}$-data with a previous time (t-1) divided by the two roots so that the resulting value was the smoothing value of the actual data as could be seen in Table 3. The smooth coefficient was symbolized by $\mathrm{Vj}$.

Table 4. Signal of wavelet and smooth

\begin{tabular}{lccccc}
\hline Time & D1 & D2 & D3 & D4 & S4 \\
\hline 1 & -234.38 & -149.66 & -119.47 & -530.17 & 21283.68 \\
2 & 242.19 & -156.49 & -248.51 & -665.44 & 21328.26 \\
3 & -234.37 & -47.12 & -347.46 & -742.65 & 21371.60 \\
. &. &. &. &. &. \\
. &. &. &. &. &. \\
. &. &. &. &. &. \\
326 & -31.25 & 272.71 & 4.50 & 690.33 & 19563.72 \\
327 & 226.56 & 216.06 & -175.00 & 715.06 & 19517.31 \\
328 & -234.37 & -123.54 & -314.60 & 710.25 & 19462.26 \\
\hline
\end{tabular}

Table 4 showed that the data decomposition using Daubechies 4 wavelet with level 4 produced 5 signals, namely wavelet signal D1 is a wavelet signal at the first level, D2 is a wavelet signal at the second level, D3 is a wavelet signal at the third level and D4 is a wavelet signal at the fourth level, while S4 is smooth at level 4. After obtaining a wavelet and smooth signal, then signal stationary testing is carried out at each level that has been generated.

\section{Stationary Test}

Signal stationary testing at each level in this study was conducted in two ways, namely identifying the plot and analyzing it with the ADF test. ADF test to determine the stationarity of the signal. Table 5 showed the ADF test for smooth signals and wavelet signals at each level.

Table 5. Statistical test results with a wavelet and smooth signal ADF test

\begin{tabular}{lccl}
\hline Signal & t statistics & P-value & Category \\
\hline First level of Wavelet & -14.08 & $<0.01$ & Stationary \\
Second level of Wavelet & -9.20 & $<0.01$ & Stationary \\
Third level of Wavelet & -4.99 & $<0.01$ & Stationary \\
Fourth level of Wavelet & -7.80 & $<0.01$ & Stationary \\
Fourth level of Smooth & -1.23 & 0.89 & Non-stationary \\
\hline
\end{tabular}

The ADF statistical test results in Table 5 showed that all wavelet signals were stationary. It could be seen from the p-value was less than 0.05 on the first level of Wavelet signal, the second level of Wavelet signal, the third level of Wavelet signal, and the fourth level of Wavelet signal so it supports to reject Ho which mean that all wavelet signals had not unit-roots. It could be concluded that the wavelet signal produced was stationary.However, the 
smooth signal had a p-value greater than 0.05 which is 0.89 so it did not support rejection of $\mathrm{H}_{0}$. The decision to reject $\mathrm{H}_{0}$ meant that the smooth signal had a root unit and it could be said that the smooth signal was not stationary. The signal could be modeled using ARIMA if it is stationary, therefore smooth signals needed to differencing until the signal became stationary and could be modeling. The statement needed to be proven by the ACF plot. Figure 3 showed the plot of the wavelet signal that is formed.

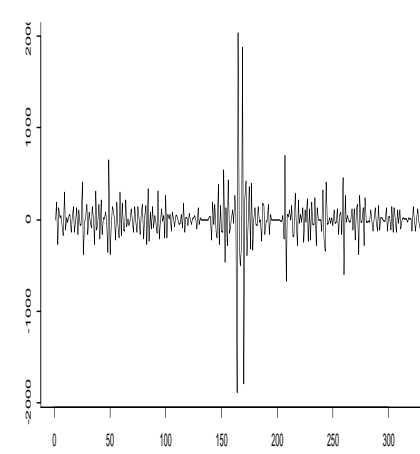

(a)

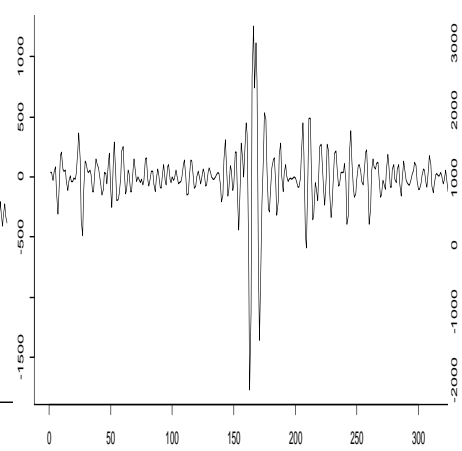

(b)

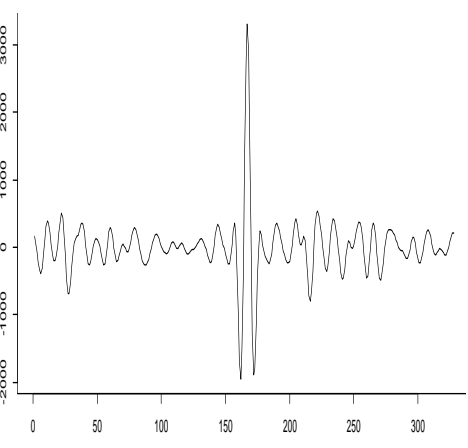

(c)

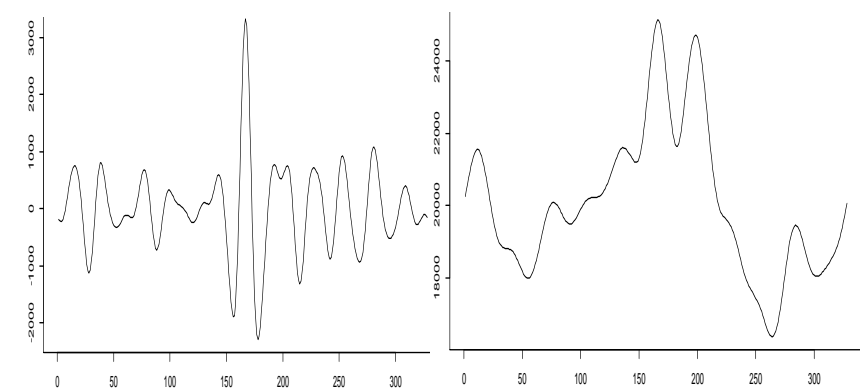

(d)

(e)

Fig. 3. Plot signal (a) first level wavelet, (b) second-level wavelet, (c) third level wavelet, (d) fourth level wavelet, (e) smooth

At a glance, it could be seen that most of the signal plots form a constant pattern of mean and variance values. Figure 3 showed the plot of a wavelet signal having a constant mean, however, the plot of a smooth signal having a non-constant mean.

\section{Modeling of MODWT signal using ARIMA}

At this stage, modeling each wavelet signal at each level with the ARMA model. The steps to model a signal are the same as the steps to model an ARMA in general, first choosing the best model from the candidate model and then modeling the signal using ARMA.

\section{First Level Wavelet Signal (D1)}

Model candidates were obtained by looking at the ACF plot, PACF plot, and ESACF.Some model candidates showed that the best ARMA $(p, q)$ model for the first level wavelet signal was ARMA $(6,2)$. Modeling using ARMA $(6,2)$ obtained an autoregressive coefficient (AR) of $\Phi_{1}$ of $-0.19, \Phi_{2}$ of $-0.25, \Phi_{3}$ of $-0.20, \Phi_{4}$ of $-0.01, \Phi_{5}$ is -0.43 , and $\Phi_{6}$ is 
0.03 while the moving average coefficient (MA) is obtained by the value of $\theta_{1}$ by -1.98 , and $\theta_{2}$ by 0.98 . The estimation model obtained formed at the first level wavelet signal with $\operatorname{ARMA}(6,2)$ is as follows:

$$
\widehat{\mathrm{D}}_{1, \mathrm{t}}=-0.19 \widehat{\mathrm{D}}_{1, \mathrm{t}-1}-0.25 \widehat{\mathrm{D}}_{1, \mathrm{t}-2}-0.20 \widehat{\mathrm{D}}_{1, \mathrm{t}-3}-0.01 \widehat{\mathrm{D}}_{1, \mathrm{t}-\mathrm{-}}-0.43 \widehat{\mathrm{D}}_{1, \mathrm{t}-5}+0.03 \widehat{\mathrm{D}}_{1, \mathrm{t}-\mathrm{-}}+\widehat{\varepsilon}_{1, \mathrm{t}}+1.98 \widehat{\varepsilon}_{1, \mathrm{t}-1}-0.98 \widehat{\varepsilon}_{1, \mathrm{t}-2}
$$

\section{Second Level Wavelet Signal (D2)}

Some model candidates showed that the best ARMA (p, q) model for the second level wavelet signal is ARMA $(5,6)$. Modeling using ARMA $(5,6)$ obtains an AR coefficient of $\Phi_{1}$ of $0.97, \Phi_{2}$ of $-1.11, \Phi_{3}$ of $0.79, \Phi_{4}$ of -0.72 , and $\Phi_{5}$ of 0.10 while the MA coefficient obtained a value of $\theta_{1}$ of $2.46, \theta_{2}$ of $0.58, \theta_{3}$ of $-2.74, \theta_{4}$ of $-1.93, \theta_{5}$ of $0.36, \theta_{6}$ of 0.43 , and mean 0.05 .The estimation model obtained is formed on the second level wavelet signal with ARMA $(5,6)$ as follows:

$$
\begin{aligned}
\widehat{\mathrm{D}}_{2, \mathrm{t}}= & 0.05+0.97 \widehat{\mathrm{D}}_{2, \mathrm{t}-1}-1.11 \widehat{\mathrm{D}}_{2, \mathrm{t}-2}+0.79 \widehat{\mathrm{D}}_{2, \mathrm{t}-3}-0.72 \widehat{\mathrm{D}}_{2, \mathrm{t}-4}+0.10 \widehat{\mathrm{D}}_{2, \mathrm{t}-5}+\varepsilon_{1, \mathrm{t}}-2.46 \varepsilon_{1, \mathrm{t}-1} \\
& -0.58 \varepsilon_{1, \mathrm{t}-2}+2.74 \varepsilon_{1, \mathrm{t}-3}+1.93 \varepsilon_{1, \mathrm{t}-4}-0.36 \varepsilon_{1, \mathrm{t}-5}-0.43 \varepsilon_{1, \mathrm{t}-6}
\end{aligned}
$$

3. Third Level Wavelet Signal (D3)

Model candidates show that the best ARMA model $(p, q)$ for the third level wavelet signal is ARMA $(12,0)$. Modeling using ARMA $(12,0)$ obtained an AR coefficient of $\Phi_{1}$ of $3.33, \Phi_{2}$ of $-4.65, \Phi_{3}$ of $3.59, \Phi_{4}$ of $-3.42, \Phi_{5}$ of $5.81, \Phi_{6}$ is $-6.89, \Phi_{7}$ is $4.66, \Phi_{8}$ is $-2.90, \Phi_{9}$ is $3.49, \Phi_{10}$ is $-3.70, \Phi_{11}$ is 2.11 , and $\Phi_{12}$ of -0.52 . The estimation model obtained formed on the third level wavelet signal with ARMA $(12,0)$ is as follows:

$$
\widehat{\mathrm{D}}_{3, \mathrm{t}}=3.33 \widehat{\mathrm{D}}_{3, \mathrm{t}-1}-4.65 \widehat{\mathrm{D}}_{3, \mathrm{t}-2}+3.59 \widehat{\mathrm{D}}_{3, \mathrm{t}-\mathrm{3}-3}-3.42 \widehat{\mathrm{D}}_{3, \mathrm{t}-4}+5.81 \widehat{\mathrm{D}}_{3, \mathrm{t}-5}-6.89 \widehat{\mathrm{D}}_{3, \mathrm{t}-\mathrm{-}}+4.66 \widehat{\mathrm{D}}_{3, \mathrm{t}-\mathrm{7}}-2.90 \widehat{\mathrm{D}}_{3, \mathrm{t}-\mathrm{8}}+3.49 \widehat{\mathrm{D}}_{3, \mathrm{t}-\mathrm{9}-}-3.70 \widehat{\mathrm{D}}_{3,}
$$

\section{Fourth Level Wavelet Signal (D4)}

Model candidates show that the best ARMA model $(p, q)$ for the fourthlevel wavelet signal is ARMA $(7,0)$.Modeling using ARMA $(7,0)$ obtained an AR coefficient of $\Phi_{1}$ of 3.44, $\Phi_{2}$ of $-4.81, \Phi_{3}$ of $4.14, \Phi_{4}$ of $-3.63, \Phi_{5}$ of $3.13, \Phi_{6}$ is -1.58 , and $\Phi_{7}$ is 0.30 . The estimation model obtained formed on the fourth level wavelet signal with ARMA $(7,0)$ is as follows:

$$
\widehat{\mathrm{D}}_{4, \mathrm{t}}=3.44 \widehat{\mathrm{D}}_{4, \mathrm{t}-\mathrm{1}}-4.81 \widehat{\mathrm{D}}_{4, \mathrm{t}-2}+4.14 \widehat{\mathrm{D}}_{4, \mathrm{t}-3}-3.63 \widehat{\mathrm{D}}_{4, \mathrm{t}-\mathrm{4}}+3.13 \widehat{\mathrm{D}}_{4, \mathrm{t}-5}-1.58 \widehat{\mathrm{D}}_{4, \mathrm{t}-\mathrm{6}}+0.30 \widehat{\mathrm{D}}_{4, \mathrm{t}-\mathrm{t}}
$$

5. Fourth Level Smooth Signal (S4)

Smooth signal had a form that is not stationary, so before it was modeled in the form of ARIMA it is necessary to do differencing before the signal becomes stationary. After being first differencing, the ADF test results obtained on the smooth signal is -8.82 with a p-value of 0.01 . Based on the ADF test, it can be shown that the smooth signal becomes stationary after being first differencing. After the signal becomes stationary, the next ARIMA model is carried out with steps like the previous signals.

Some model candidates show that the best ARIMA model $(\mathrm{p}, \mathrm{d}, \mathrm{q})$ for smooth signals was ARIMA $(3,1,0)$. Modeling using ARIMA $(3,1,0)$ obtained an autoregressive coefficient (AR) that is " $\Phi_{1}$ of $2.84, \Phi_{2}$ of -2.72 , and $\Phi_{3}$ of 0.88 . The estimation model that is formed from the coefficients " $\Phi$ " and $\theta$ for smooth signals is as follows:

$$
\widehat{\mathrm{S}}_{4, \mathrm{t}}=3.84 \widehat{\mathrm{S}}_{4, \mathrm{t}-1}-5.73 \widehat{\mathrm{S}}_{4, \mathrm{t}-2}+3.60 \widehat{\mathrm{S}}_{4, \mathrm{t}-\mathrm{z}}-0.88 \widehat{\mathrm{S}}_{4, \mathrm{t}-4}
$$

\section{MODWT-ARMA Model}


The MODWT-ARMA model obtained by combining the alleged model of all signals, both wavelet and smooth signals using equation (8), is shown as follows:

$$
\widehat{\mathrm{Y}}_{\mathrm{t}}=\widehat{\mathrm{D}}_{1, \mathrm{t}}+\widehat{\mathrm{D}}_{2, \mathrm{t}}+\widehat{\mathrm{D}}_{3, \mathrm{t}}+\widehat{\mathrm{D}}_{4, \mathrm{t}}+\widehat{\mathrm{S}}_{4, \mathrm{t}}
$$

where $\widehat{D}_{1, t}$ is the estimated value of the first level wavelet signal at the t-time, $\widehat{D}_{2, t}$ is the estimated value of the second level wavelet signal at the t-time, $\widehat{D}_{3, t}$ is the estimated value of the third level wavelet signal at the t-time, $\widehat{\mathrm{D}}_{4, \mathrm{t}}$ is the estimated value of the fourth level wavelet signal at the t-time, $\widehat{S}_{4, t}$ is the estimated value of the fourth level smooth signal at the t-time.

$$
\begin{aligned}
& \widehat{\mathrm{Y}}_{\mathrm{t}}=0.05-0.19 \widehat{\mathrm{D}}_{1, \mathrm{t}-1-1}-0.25 \widehat{\mathrm{D}}_{1, \mathrm{t}-2-2}-0.20 \widehat{\mathrm{D}}_{1, \mathrm{t}-3}-0.01 \widehat{\mathrm{D}}_{1, \mathrm{t}-4}-0.43 \widehat{\mathrm{D}}_{1, \mathrm{t}-\mathrm{-}-\mathrm{t}}+0.03 \widehat{\mathrm{D}}_{1, \mathrm{t}-\mathrm{t}-6} \\
& +0.97 \widehat{\mathrm{D}}_{2, \mathrm{t}-\mathrm{t}}-1.11 \widehat{\mathrm{D}}_{2, \mathrm{t}-2}+0.79 \widehat{\mathrm{D}}_{2, \mathrm{t}-3}-0.72 \widehat{\mathrm{D}}_{2, \mathrm{t}-4}+0.10 \widehat{\mathrm{D}}_{2, \mathrm{t}-\mathrm{-}-\mathrm{t}}+3.33 \widehat{\mathrm{D}}_{3, \mathrm{t}-\mathrm{t}-1}-4.65 \widehat{\mathrm{D}}_{3, \mathrm{t}-2} \\
& +3.59 \widehat{\mathrm{D}}_{3, \mathrm{t}-\mathrm{-}-3}-3.42 \widehat{\mathrm{D}}_{3, \mathrm{t}-\mathrm{t}}+5.81 \widehat{\mathrm{D}}_{3, \mathrm{t}-\mathrm{-}}-6.89 \widehat{\mathrm{D}}_{3, \mathrm{t}-\mathrm{6}}+4.66 \widehat{\mathrm{D}}_{3, \mathrm{t}-\mathrm{T}}-2.90 \widehat{\mathrm{D}}_{3, \mathrm{t}-\mathrm{-}}+3.49 \widehat{\mathrm{D}}_{3, \mathrm{t}-\mathrm{9}} \\
& \text { - } 3.70 \widehat{\mathrm{D}}_{3, \mathrm{t}-10}+2.11 \widehat{\mathrm{D}}_{3, \mathrm{t}-11}-0.52 \widehat{\mathrm{D}}_{3, \mathrm{t}-12}+3.44 \widehat{\mathrm{D}}_{4, \mathrm{t}-1}-4.81 \widehat{\mathrm{D}}_{4, \mathrm{t}-2}+4.14 \widehat{\mathrm{D}}_{4, \mathrm{t}-\mathrm{3}}-3.63 \widehat{\mathrm{D}}_{4, \mathrm{t}-4} \\
& +3.13 \widehat{\mathrm{D}}_{4, \mathrm{t}-\mathrm{-}-1}-1.58 \widehat{\mathrm{D}}_{4, \mathrm{t}-\mathrm{6}}+0.30 \widehat{\mathrm{D}}_{4, \mathrm{t}-\mathrm{-}}+3.84 \widehat{\mathrm{S}}_{4, \mathrm{t}-1}-5.73 \widehat{\mathrm{S}}_{4, \mathrm{t}-2}+3.60 \widehat{\mathrm{S}}_{4, \mathrm{t}-3}-0.88 \widehat{\mathrm{S}}_{4, \mathrm{t}-4} \\
& +\hat{\varepsilon}_{1, \mathrm{t}}+1.98 \hat{\varepsilon}_{1, \mathrm{t}-1}-0.98 \hat{\varepsilon}_{1, \mathrm{t}-2}+\varepsilon_{1, \mathrm{t}}-2.46 \varepsilon_{1, \mathrm{t}-1}-0.58 \varepsilon_{1, \mathrm{t}-2}+2.74 \varepsilon_{1, \mathrm{t}-3}+1.93 \varepsilon_{1, \mathrm{t}-4} \\
& -0.36 \varepsilon_{1, \mathrm{t}-5}-0.43 \varepsilon_{1, \mathrm{t}-6}
\end{aligned}
$$

where $\widehat{\varepsilon}_{1, \mathrm{t}}$ is the estimated error of the first level wavelet signal at the t-time, and $\hat{\varepsilon}_{2, \mathrm{t}}$ is the estimated error of the second level wavelet signal at the t-time.

\subsection{Test of Goodness Fit of The Model}

The criterion for model goodness is the measurement of a model that is suitable for data. A good model will affect the accuracy of data predictions. In this study, error assessment of data using the RMSE and normalized error values. The normalized error was symbolized by Q. The result of error assessment was as follows:

Table 6. RMSE and Q values in the MODWT-ARMA and ARIMA models

\begin{tabular}{llrc}
\hline Data & Model & RMSE & $Q$ \\
\hline Training & ARIMA & 731.99 & 0.09 \\
& MODWT-ARMA & 96.46 & 0.00 \\
Testing & ARIMA & 2365.85 & 2.77 \\
& MODWT-ARMA & 1175.97 & 0.68 \\
\hline
\end{tabular}

The RMSE value and Q value in Table 6 showed that the MODWT-ARMA model had smaller $\mathrm{Q}$ and RMSE values than the ARIMA model. In training data, MODWT-ARMA had the values of $\mathrm{Q}$ and RMSE are 0.00 and 96.46 while the values of $\mathrm{Q}$ and RMSE of the ARIMA model were 0.09 and 731.99. In test data, MODWT-ARMA had the values of $Q$ and RMSE are 0.68 and 1175.97 while the values of Q and RMSE of the ARIMA model were 2.77 and 2365.85. So based on testing the goodness of the criteria of models, it can be concluded that the modeling of daily broiler chicken data in Bogor in 2018 using MODWT-ARMA is better than the ARIMA model.

\section{Conclusion}


Increasing and decreasing chicken prices in an uncertain market make the chicken price data fluctuate so that sometimes has a trend. Moreover, increasing demand for chicken at a certain season like Idul Fitri and other big days makes the data shape have wave patterns that cause the data is not stationary. In this study, broiler chicken price data in Bogor in 2018 are better modeled using the MODWT-ARMA model than the ARIMA model. Data modeling using MODWT-ARMA has a smaller error value compared to using ARIMA.

The conclusion that can be obtained in this study is the modeling of data using the MODWT-ARMA model both for use in time-series data that has fluctuations and not stationary and modeling using the MODWT-ARMA model can also improve the accuracy of predictions of data compared to using the ARIMA model in the case study of broiler chicken price data in Bogor in 2018.

\section{References}

[1] Agustini, K.:PerbandinganMetodeTransformasi $\quad$ Wavelet sebagaiPraprosespadaSistemIdentifikasiPembicara. InstitutPertanian Bogor, ID (2006)

[2] Akansu, A.N., Haddad, R.A.: Multiresolution Signal Decomposition Transforms, Subbands, and Wavelets Second Edition. Academic Press, US (2001)

[3] Burnham, K.P., Anderson, D.R.: Model Selection and Multimodel Inference. Springer, US (2002)

[4] Chai, T., Draxler, R.R.: Root Mean Square Error (RMSE) or Mean Absolute Error (MAE)? Arguments against avoiding RMSE in the literature. Geoscientific Model Development. 7 (3):1247 (2014).

[5] Farima,V.Z., Utami, H.: Indonesia dengan Model Maximal Overlap Discrete. JurnalStatistikaUniversitasMuhammadiyah Semarang. 6(1) (2018)

[6] Juanda, B., Junaidi.: EkonometrikaDeretWaktu. IPB Press, ID (2012)

[7] Kurnia, M.T., Nugrahani, E.H., Sumarno, H.:Analisis Wavelet danArimauntukPeramalanHargaEmas Pt. AntamTbk Indonesia. Journal of Math and Its Application. 13(2) 11 (2014)

[8] Konishi, S., Kitagawa, G..:Information Criteria and Statistical Modeling. Kyushu University, JP (2008)

[9] Mondal, D., Percival, D.B.: M-estimation of Wavelet Variance. Ann. Inst. Stat. Math. 64(1) 27$53(2012)$

[10] Nason, G.:Wavelet Methods in Statistics with R. Journal of the Royal Statistical Society: Series A (Statistics in Society). 173 (2009)

[11] Percival, D.B., Walden, A.T.: Wavelet Method for Time Series Analysis. Cambridge University, US (2000)

[12] Renaud, O., Starck, J.L., Murtagh, F.: Prediction Based on a Multiscale Decomposition. Int. J. Wavelets, Multiresolution Inf. Process. 01(02) 217-232 (2003)

[13] Sadik, K., Erfiani., Noviyanita, W.P.: PendeteksianPencilanAditifdanInovatifdalam Data DeretWaktumelaluiMetodeIteratif. Forum StatistikadanKomputasi - ISSN: 0853-8115. 13 (2) (2008) [14] Schurer, J.: Application of Wavelet Variance Analysis to Stock Prices in Select Technology Companies. Czech Technical University, CZ (2017)

[15] Stebler, Y., Guerrier, S., Skaloud, J., Victoria-Feser, M.P.: Generalized Method of Wavelet Moments for Inertial Navigation Filter Design. IEEE Trans. Aerosp. Electron. Syst. 50(3) 22692283 (2014)

[16] Suma'inna, S., Gumilar, G.:ImplementasiTransformasi Wavelet DaubechiespadaKompresi Citra Digital. Cauchy. 2(4) 211 (2013)

[17] Walker, J.S.: A Primer on Wavelets and Their Scientific Applications Second Edition. University of Wisconsin, US (2008) 
[18] Warsito, B., Subanar., Abdurakhman.:Pemodelan Time Series dengan Maximal Overlap Discrete Wavelet Transform. Pros. Semin. Nas. Stat. (1998). 605-613 (2013)

[19] Wei, W.W.S.: Time Series Analysis Univariate and Multivariate Methods Fourth Edition. Pearson Education Inc, US (2006)

[20] Zhu L, Wang Y, Fan Q.: MODWT-ARMA Model for Time Series Prediction. Appl. Math. Model. 38 (5-6) 1859-1865 (2014) 